\title{
Calibrating Dynamic Pedestrian Route Choice with an Extended Range Telepresence System
}

\author{
Tobias Kretz, Stefan Hengst, Vidal Roca, \\ PTV Planung Transport Verkehr AG \\ Stumpfstr. 1, D-76131 Karlsruhe \\ \{Firstname. Lastname $\}$ eptv.de \\ Antonia Perez Arias, Simon Friedberger, and Uwe D. Hanebeck \\ Institute for Anthropomatics \\ Chair for Intelligent Sensor-Actuator-Systems \\ Karlsruhe Institute of Technology \\ Kaiserstrasse 12, D-76128 Karlsruhe \\ \{Firstname. Lastname\}akit.edu
}

\begin{abstract}
In this contribution we present the results of a pilot study in which an Extended Range Telepresence System is used to calibrate parameters of a pedestrian model for simulation. The parameters control a model element that is intended to make simulated agents walk in the direction of the estimated smallest remaining travel time. We use this to, first, show that that an Extended Range Telepresence System can serve for such a task in general and second to actually find simulation parameters that yield realistic results.
\end{abstract}

\section{Introduction}

\subsection{Desired Walking Direction of Pedestrians}

The Social Force Model [20,9] is a model to simulate the dynamics of crowds of pedestrians ${ }^{1}$. It was first introduced in 1995 [10] and has been actively discussed since. The modeling approach is derived from Newtonian Dynamics where forces between objects determine the acceleration of an object. From the acceleration speed and location changes follow by integration. In the Social Force Model there are three sources for the total force acting on a pedestrian agent: a force "pulling" a pedestrian (or an agent respectively) toward his destination, forces between pedestrians (usually repulsive), and forces repelling the agent from walls and obstacles.

For our purposes details of the repulsive forces between

\footnotetext{
${ }^{1}$ For an overview of related work on models for pedestrian dynamics see [43]
}

pedestrians and other pedestrians/walls/obstacles are irrelevant and we focus on the driving force term

$$
\vec{f}_{i}^{\text {drive }}(t)=\frac{v_{i}^{0}(t) \cdot \hat{v}_{i}^{0}(t)-\vec{v}_{i}(t)}{\tau}
$$

which makes pedestrian agent $i$ head toward his desired destination. $\hat{v}_{i}^{0}(t)$ is the direction of the desired velocity and $v_{i}^{0}(t)$ the desired speed. $\vec{v}_{i}(t)$ is the current velocity. $\tau$ is a model parameter, which can be interpreted as summarizing the effects of reaction time and inertia.

The traffic simulation software VISSIM in version 5.30 includes a pedestrian simulation built on the Social Force Model $[31,4,39]$. It allows calculation of the desired direction $v_{i}^{0}(t)$ either as pointing in the direction of the shortest path (considering obstacles) or pointing in the direction of the "estimated smallest remaining travel time". This second method - called "dynamic potential" - is controlled using two parameters $g$ and $h$. By default these are set to $g=1.5$ and $h=0.6$ in VISSIM 5.30 as it is installed. Recently details of the method have been published [30]. As there is nearly no empirical data available that can be used to calibrate parameters $g$ and $h$, so far they have been set to accommodate expectations on the visual impression ${ }^{2}$. While clearly using the dynamic potential method yields better results in such situations than when each agent moves predominantly into the direction of shortest path the need to collect data is obvious.

\footnotetext{
${ }^{2}$ Compare for example simulation: www.youtube.com/ user/ptvvision\#p/u/3/8SmRBTJ-jeU, vs. observation: www. youtube.com/watch?v=49HIZbFLPhg www. youtube. $\mathrm{com} /$ watch?v=jtKkHJXUVQY, www. youtube. com/watch?v= LodYbDco0 jY, www . youtube. com/watch?v=1WqnQjwAAac.
} 
The following is a summary of the method to calculate the direction of the estimated smallest remaining travel time (dynamic potential):

First a field $f$, which is defined on a regular grid with spacing of about $20 \mathrm{~cm}$, is calculated for a particular destination. A field value is either $f(x, y)=1$, if the location is not occupied by an agent or $0<f<1$, if it is occupied. The precise value of $f$ depends on the movement direction of the location occupying agent: if that particular agent is heading (spatially) towards the corresponding destination then the value of $f$ is larger (closer to 1 ) than if the agent moves away from it. Parameter $g$ sets the overall impact of the presence of an agent (i.e. if $g=0$ then $f=1$ everywhere) and parameter $h$ determines the impact of the movement direction of the agent (i.e., if $h=0$ then each occupied grid point has a value $f=1 /(1+g)$.

The full equation for $1 / f(x, y)$ if a grid point is occupied by an agent is

$$
\frac{1}{f(x, y)}=1+\max \left(0, g\left(1+h \frac{\vec{v}(x, y) \cdot \nabla S(x, y)}{\left\langle v^{0}\right\rangle|\nabla S(x, y)|}\right)\right)
$$

with $-\nabla S(x, y)$ being the direction of the shortest path, $\left\langle v^{0}\right\rangle$ being the average of desired speeds of agents who are heading for that destination, $\vec{v}(x, y)$ the velocity of the location occupying agent.

Once the field $f(x, y)$ is calculated, it is numerically integrated solving the Eikonal equation

$$
|\nabla T(x, y)|^{2}=\frac{1}{f(x, y)^{2}}
$$

with $T(x, y)$ being the desired field of estimated remaining travel time. For the integration method see [22, 17, 18, 19]

The desired walking direction for an agent $i$ at a location $(x, y)$ at time $t$ heading towards the corresponding destination results from this as

$$
\hat{v}_{i}^{0}(t)=-\frac{\nabla T(x, y, t)}{|\nabla T(x, y, t)|}
$$

It is hoped that the telepresence system can assist in shedding light on this topic by allowing to supplement real participants with simulated ones.

\subsubsection{Related work}

Route choice issues of pedestrians are an open issue of discussion. This is mainly due to the fact that the environment in which pedestrians are moving usually is highly complex [6] and that often many pedestrians are involved. It has been stressed that from an application point of view a realistic simulation of situations where smallest travel time is balanced against shortest path is highly relevant while at the same time available models usually do not offer good solutions [40]. Travel time as determinant of the motion has been discussed and modeled in a number of previous works, sometimes for macro- or mesoscopic approaches $[13,14,32,8,44]$, but more often within the scope of microscopic models. Of these the majority are field-based as the dynamic potential approach $[12,49,46,26,27,23,47$, $29,28,7,24$ ], but there exist also other methods which are based on an additional routing network, discrete choice or heuristics [5, 3, 11, 21, 34].

\subsection{Extended Range Telepresence}

Telepresence systems aim at creating the impression of being present in a (remote real or virtual) target environment. The feeling of presence is achieved by visual and acoustic sensory information recorded from the target environment and presented to the user on an immersive display. Furthermore, in extended range telepresence the user's motion is tracked and transferred to his virtual representation, so that the user can freely move and walk around in the target environment by using his feet instead of devices like joysticks, foot pedals, steering wheels $[1,48]$ or similar input devices. As a result, by using extended range telepresence, the user can use his proprioception, i.e., his own sense of motion, which is especially important for way-finding in target environments [2].

Mechanical locomotion interfaces that allow natural walking of the user in the target environment include passive devices like the commercially available VirtuSphere ${ }^{3}$, where the user walks inside a large sphere, omnidirectional treadmills [15, 45], or actively driven floor surfaces [16, 50]. However such interfaces require expensive mechanical setups and complex control algorithms.

To allow exploration of an arbitrarily large target environment while moving in a limited user environment, we use Motion Compression [35, 41]. While preserving the length of the path and the turning angles, Motion Compression curves the path in the target environment until it fits into the limited user environment and then guides the user on this user path. At the same time, the user has the impression of walking on the original target path, since humans do not realize small inconsistencies between the displayed and the perceived path curvature during locomotion. Experimental results in [35] show that test persons are no longer able to detect the direction of curvature for curvatures $\kappa<0.1 \mathrm{~m}^{-1}$ and subjects quickly accustom to the inconsistency of visual and proprioceptive perception even for a user environment of 3 by $3 \mathrm{~m}$ where the curvature is usually greater than $0.3 \mathrm{~m}^{-1}$. The available user environment in our system is 4 by $4 \mathrm{~m}$. Figure 1 shows exemplary trajectories that a user would cover in both environments.

\footnotetext{
${ }^{3}$ (http://www.virtusphere.com)
} 
For this project the environment displayed to the user of the telepresence is 3D animation output of VISSIM as explained in $[38,37]$. In this way the telepresence user moves through an environment with a number of virtual agents. These virtual (simulated) agents react to the telepresence user as if he was a simulated agent. This means that the telepresence user has virtually stepped into the pedestrian simulation. Figure 2 shows the user interface of the combined system. Through the egocentric view and the possibility of walking freely in the simulation, the user is not passively looking at the simulation, but he feels present in the simulation and can interact with other pedestrians.
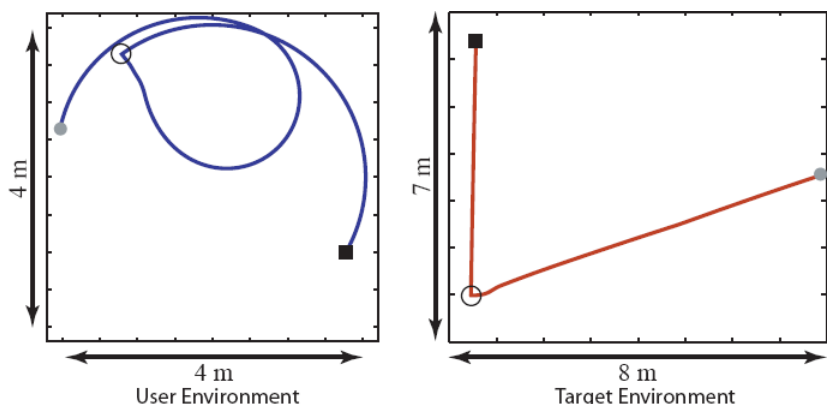

Figure 1. A trajectory as the telepresence user has actually walked (left) and as he believes he has walked (right). The transformation process is called Motion Compression.

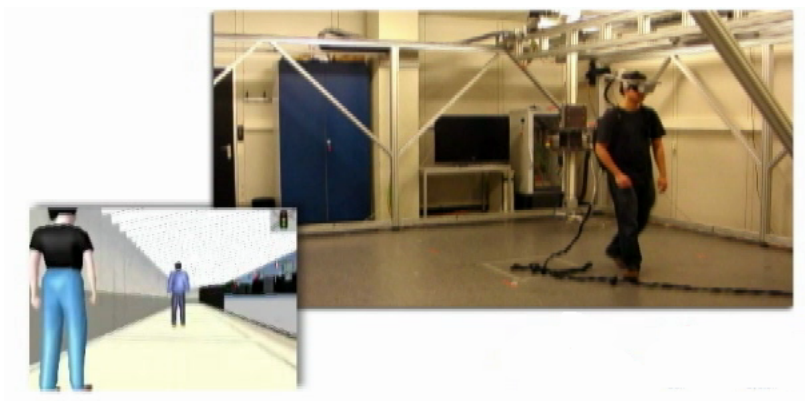

Figure 2. A user in the telepresence system (upper right) and what is shown to him via the visual unit (lower left).

\section{Experiment Description and Task Formula- tion}

For the scenario as shown in Figures 3 and 4 the user of the telepresence is told that his task is to walk from his starting position to a destination on the other side of the wall when the traffic light turns green such that he arrives as early as possible. The wall is $12.5 \mathrm{~m}$ away from the starting position and the doors are $7.5 \mathrm{~m}$ apart (with their inner sides). The width of the doors is $1.4 \mathrm{~m}$.

The traffic light turns green in second 90 of the simulation. At that time it is estimated, if a simulated agent would

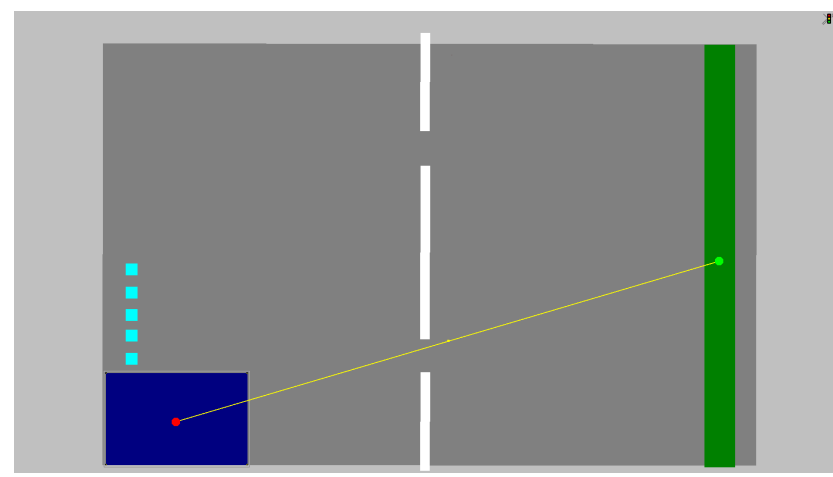

Figure 3. Top view of the scenario used for investigation of parameters $g$ and $h$. Walkable areas are shown in dark gray, obstacles in white. Simulated agents are set into the simulation on the dark blue area and follow the route as shown (red dot via yellow line to green dot) to the dark green area to the right in the way that they first head for the area with the blue and then the area with the green dot. On one of the cyan areas the telepresence user is set into the simulated environment and therefore sees at his starting position what is shown in Figure 4.

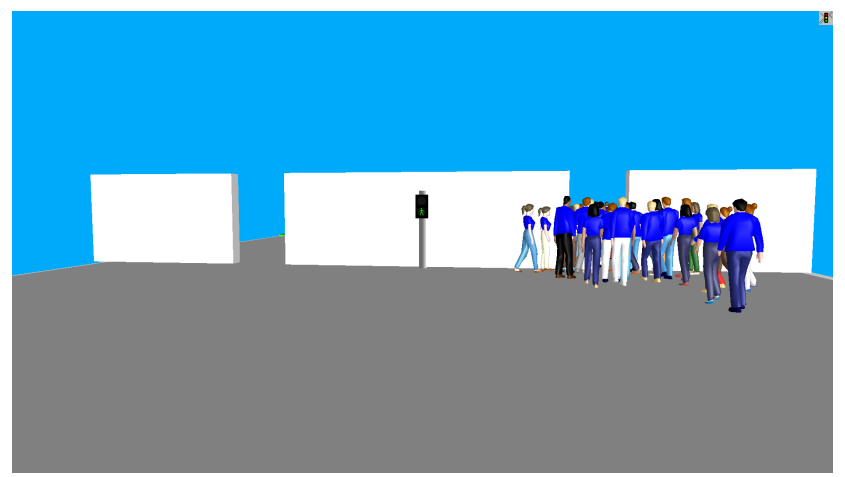

Figure 4. User view as shown to the telepresence user (the participant of the experiment) at his starting position. At first the traffic light is red. The user is told to choose one of the two doors when the light turns green so that he will arrive at the destination on the other side as soon as possible.

use the left or the right door from the gradient of field $T$. This can be visualized as following the gradient field arrows from the starting position of the user toward the destination on the other side of the wall. In a strict sense it is not guaranteed that a simulated agent will really end up at the door indicated at that point in time, as the dynamic floor field changes over time, however for this work we stick with this simplification. For evaluation this door choice of a simulated agent is compared with the actual user choice.

The participants were faced with four situations of simulated demand (the number of blue agents in Figure 4): none, few, capacity, jammed. To produce these at first it was measured how large the capacity of the door is with the chosen parameters $\left(j_{\text {cap }}=1.32\right.$ agents per second). Setting "none" as demand is obviously trivial. To have "few" demand the 

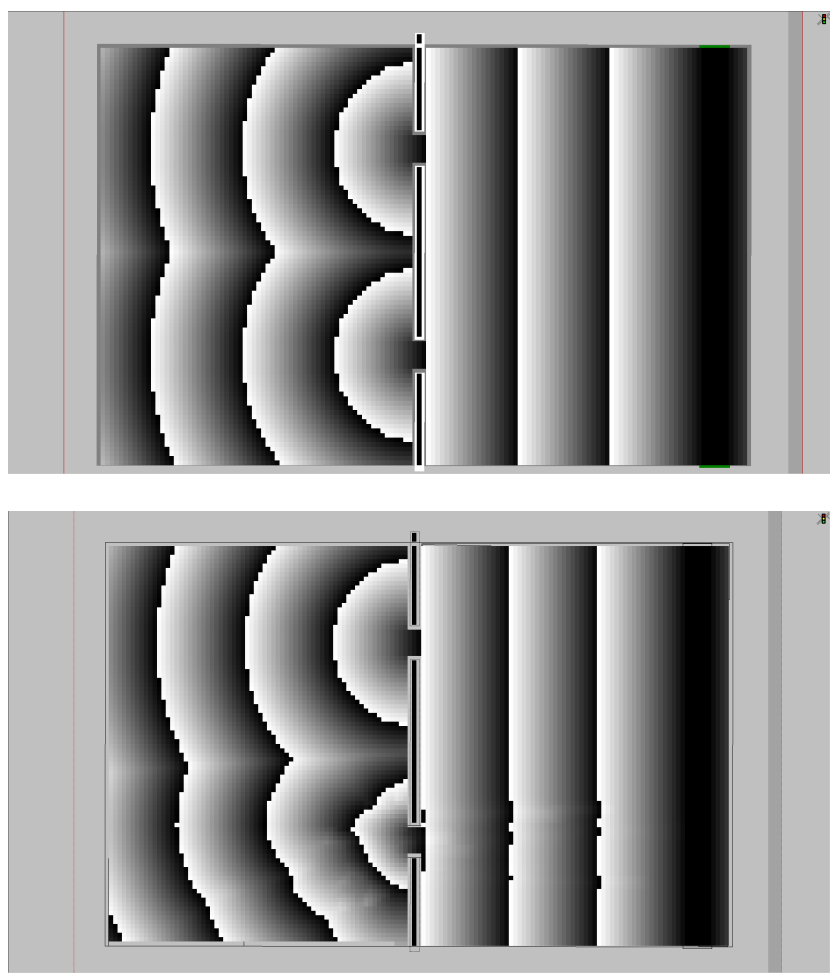

Figure 5. Field $S$ (static potential, upper figure) and field $T$ (dynamic potential, lower figure) for a certain point in time of the simulation. Generally the values of distance and estimated remaining travel time grow from right to left and with the brightness of the spot. Distance and travel time is displayed modulo the maximum brightness. See Figure 6 for the gradients' corresponding desired directions resulting from this field $T$.

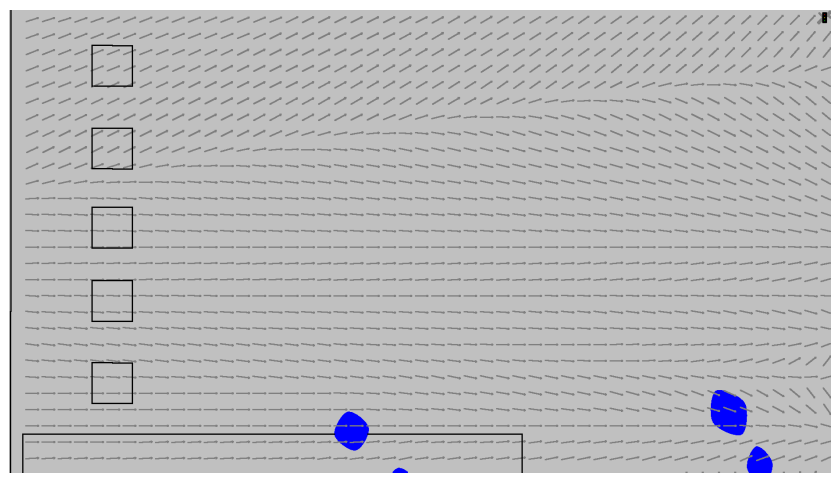

Figure 6. Desired directions for the region where the user of the telepresence (the participant) is set into the simulation. It can clearly be seen that the desired directions on some of the five starting areas point to the upper (left) door although the shorter path to the destination on the right would lead through the lower (right) door. This would be different if the desired directions were calculated under a shortest path movement paradigm.

input volume to the simulation (with respect to the number of blue agents) was well below capacity $\left(j_{\text {few }}=0.25\right.$ agents per second). For "capacity" demand exactly the mea-

\begin{tabular}{c|cccc} 
& none & few & capacity & jammed \\
\hline$-0.5 \mathrm{~m}$ & 1 & 8 & 17 & 20 \\
$-1.5 \mathrm{~m}$ & 0 & 5 & 13 & 20 \\
$-2.5 \mathrm{~m}$ & 1 & 2 & 17 & 20 \\
$-3.5 \mathrm{~m}$ & 0 & 2 & 12 & 20 \\
$-4.5 \mathrm{~m}$ & 0 & 1 & 12 & 20
\end{tabular}

Table 1. Number of participants who have chosen to walk through the left door depending on the y coordinate of starting position and demand volume of simulated agents.

sured volume $j_{\text {cap }}$ is put into the simulation right from second 0 ; and to have a "jammed" situation an input volume of $j_{\text {jammed }}=5.0$ agents per second was configured for the initial phase and then the input volume was reduced to $j_{\text {cap }}$.

The starting positions (the cyan squares) in Figure 3 were $0.5,1.5,2.5,3.5$, and $4.5 \mathrm{~m}$ to the right of the symmetry axis (the line equally far away from both doors), i.e. the $\mathrm{y}$ coordinates of the starting positions were $y=-0.5 \mathrm{~m}$, $y=-1.5 \mathrm{~m}, y=-2.5 \mathrm{~m}, y=-3.5 \mathrm{~m}$ or $y=-4.5 \mathrm{~m}$ respectively. In this way there are 20 variants of the scenario: five starting positions times four demand volume variants.

Note: Parameter $\left\langle v^{0}\right\rangle$ of equation 2 has been set to a value of $\left\langle v^{0}\right\rangle=1.3 \mathrm{~m} / \mathrm{s}$ for the work presented in this paper. In principle the ratio $h /\left\langle v^{0}\right\rangle$ could replace $h$ as investigated parameter, but as $\left\langle v^{0}\right\rangle$ is dictated by circumstances and usually takes very similar values between 1.3 and $1.7 \mathrm{~m} / \mathrm{s}$, we chose to stick with $h$ only. Therefore parameters $h$ and $g$ are by far the most relevant ones to determine if a simulated agent chooses the left or the right door. Other parameters especially those of the Social Force Model - need not to be considered.

\section{Results}

\subsection{Experiments}

We introduced 20 participants to the scenario. All of them participated in all 20 variants. As a consequence of limited available space, we refrain from documenting the decisions of all participants for all variants and give only a summary. Table 1 shows how many participants in each variant chose to walk through the left door (the door which is never used by simulated blue agents).

Table 1 shows a number of things: First, obviously one participant chose to walk the longer way when no other agent was present. Beyond that there were 11 occasions when a participant walked through the left door, when he or she had walked through the right door from a starting position more to the left, and it happened once that someone walked - from the same starting position - through the left door at "few" demand and through the right door at capacity. Naturally a model resting on the assumption on movement into the direction of smallest expected travel time cannot reproduce such behavior. The decisions of 12 of the 20 
participants were in agreement with this assumption.

Second, the case of the jammed right door and some of the cases without simulated competing demand were the only ones where all participants agreed in their decision. This implies that there cannot be one "true" parameter choice for $(g, h)$, but that in principle these are individual parameters. While this is by all means an expected result, it complicates the evaluation of the experiment.

\subsection{Simulations}

Based on the default parameter settings as delivered with the installation 26 combinations of $g$, $h$ were selected to be simulated and compared with the participants' results. For $g$ the chosen values were $0.5,1.0,1.5,2.0,2.5$ and for $h$ the values were $0.0,0.5,0.75,1.0,1.5$. It was also verified that with $g=0.0$ all simulated agents would walk through the right door from all starting positions and independently of the value of parameter $h$.

All simulations were in agreement with the assumption of smallest remaining travel time movement direction, i.e. in the simulation results no phenomena as described in the preceding subsection can be found. Again for the matter of limited space we refrain from giving all 400 single results here.

\subsection{Evaluation: Comparison}

As mentioned before, the decisions of the participants varied especially for the cases of "few" and "capacity" demand. Assuming that a hypothetical distribution of the individually correct $(g, h)$ combinations is single peaked with the peak near the averages of $g$ and $h$, we try to find parameter combinations which are best, or at least good, on average.

To do so there are three possibilities:

Method A: If a parameter combination $(g, h)$ is in agreement with one single decision of one single participant the combination receives a positive point, else a negative one. The best parameter combination is the one with the most points (with a maximum of 400 points for 20 participants and 20 decisions each).

Method B: For each participant it is checked which parameter combination has the best agreement with his or her decisions. This combination receives a point. If more than one set of parameters describe the choice equally well, all receive a point, no matter how many there are. The best parameter combination is the one with the most points (with a maximum of 20 for 20 participants).

Method C: All parameter combinations are compared to the majority choice as shown in Table 1 . The best parameter combination is the one which has most agreements (with a maximum of 20 for 20 scenario variants). Compared to method A it is therefore neglected how clear the majority decision is.

\begin{tabular}{l|cccccc}
$h \cdot g$ & 0.0 & 0.5 & 1.0 & 1.5 & 2.0 & 2.5 \\
\hline 0.0 & & & & $\mathrm{~A} / \mathrm{B} / \mathrm{C}$ & $\mathrm{A} / \mathrm{C}$ & $\mathrm{A} / \mathrm{C}$ \\
0.5 & & & & $\mathrm{~A} / \mathrm{B} / \mathrm{C}$ & $\mathrm{A} / \mathrm{C}$ & $\mathrm{A} / \mathrm{C}$ \\
0.75 & & & & & & $\mathrm{~A} / \mathrm{C}$ \\
1.0 & & & & & & $\mathrm{~A} / \mathrm{C}$ \\
1.5 & & & & & &
\end{tabular}

Table 2. This table summarizes for the three different evaluation methods which parameter combinations prove to be best reproducing participants' exit choice.

Applying method A the following eight combinations emerge as equally good (with 302 points): $(g=1.5 /$ $h=0.0),(g=1.5 / h=0.5),(g=2.0 / h=0.0)$, $(g=2.0 / h=0.5),(g=2.5 / h=0.0),(g=2.5 /$ $h=0.5),(g=2.5 / h=0.75)$, and $(g=2.5 / h=1.0)$. See also Table 2.

According to method B $(g=1.5 / h=0.0)$ and $(g=1.5$ $/ h=0.5$ ) describe best the behavior of most (namely 12 of the 20) participants. This is a subset of the best parameter choice according to method $\mathrm{A}$. The remaining best choices according to method A all describe the behavior of 11 participants and therefore rank second best according to method B. All other parameter choices describe the behavior of 6 participants. Only for three participants all choices are reproduced by one or more parameter sets.

Applying method $\mathrm{C}$ yields the same results as method $\mathrm{A}$ in terms of best parameter combinations: all of them reproduce all majority decisions correctly.

The results are summarized in Table 2 .

\subsection{Discussion}

The limitations of the proposed approach are threefold:

1. The number of 20 participants is at the lower limit of what allows to draw conclusions.

2. The investigation presented is limited to one single scenario. Other scenarios might favor other parameter choices.

3. The choice of variants (starting position and demand) has an impact on the results.

Having these limitations in mind we note the following:

- The telepresence system can be used for investigation of certain parameters of pedestrian dynamics and therefore calibration of parameters of a pedestrian dynamics simulation. For the simulated agents (blue shirt in Figure 4) that formed the jam no real persons had to be recruited.

- The choices of the participants varied significantly. It would therefore be desirable to have a distribution of 
$(g, h)$, i.e. more than one dynamic potential instead of just one.

- All three methods of evaluation yield about the same parameter sets.

- Among these best parameter sets is also $(g=1.5 /$ $h=0.5$ ) which is the one closest to the default configuration delivered with the setup $(g=1.5 / h=0.6)$.

- Further parameter sets with best agreement tend to have a larger $g$.

- All best parameter sets have at least a value of $g=1.5$ showing that introducing the method of the dynamic potential was a step in the right direction compared to the conventional routing along the shortest path which results from $g=0.0$.

As this scenario is about a discrete choice ("left or right door?") the resulting parameters should fit best for simulations that include similar discrete choices. The result cannot necessarily be transferred to situations with a continuous choice on quickest vs shortest path (e.g. when someone walks around a corner within a large group) or situations with a significant and relevant counterflow. For the first slightly smaller values of $g$ might prove to yield most realistic results, while for the latter larger values of $h$ might be necessary. However, without empirical data available this is just a guess.

\section{Summary}

It has been demonstrated with a concrete example that it is possible to use a telepresence system to verify the parameter choice of a pedestrian dynamics simulation model. Compared to a real experiment the telepresence system saved the resources which would have been required to use real people instead of the simulated agents causing the delays.

At the same time it has been shown - admittedly on a rather coarse grain level - that within the pedestrian dynamics simulation the concept of the dynamic potential and as a detail equation 2 improves the degree of realism concerning the choice behavior of simulated agents in this particular scenario.

\section{Acknowledgments}

This project was partially funded by the Bundesministerium für Wirtschaft und Technologie (German Federal Ministry of Economics and Technology) due to a decision of the Deutscher Bundestag (German Parliament). The authors thank Jochen Görtler for assisting in the evaluation process of the experiment and all participants of the experiment.

\section{References}

[1] C. Bunz, M. Deflorian, C. Hofer, F. Laquai, M. Rungger, F. Freyberger, and M. Buss. Development of an Affordable Mobile Robot for Teleexploration. In Proceedings of IEEE Mechatronics \& Robotics (MechRob'04), 2004. 2

[2] R. P. Darken, T. Allard, and L. B. Achille. Spatial Orientation and Wayfinding in Large-Scale Virtual Spaces: An Introduction. Presence, 7(2):101-107, 1998. 2

[3] D. Dressler, M. Groß, J. Kappmeier, T. Kelter, J. Kulbatzki, D. Plümpe, G. Schlechter, M. Schmidt, M. Skutella, and $\mathrm{S}$. Temme. On the use of network flow techniques for assigning evacuees to exits. Procedia Engineering, 3:205-215, 2010. 2

[4] M. Fellendorf and P. Vortisch. Microscopic Traffc Flow Simulator VISSIM. In J. Barceló, editor, Fundamentals of Traffic Simulation, pages 63-94. Springer, 2010. 1

[5] P. Fiorini and Z. Shiller. Motion planning in dynamic environments using velocity obstacles. The International Journal of Robotics Research, 17(7):760, 1998. 2

[6] F. Gräßle and T. Kretz. An Example of Complex Pedestrian Route Choice. In Peacock et al. [36], pages 767-771. 2

[7] S. Guy, J. Chhugani, S. Curtis, P. Dubey, M. Lin, and D. Manocha. PLEdestrians: A Least-Effort Approach to Crowd Simulation. In M. Otaduy and Z. Popovic, editors, Eurographics/ ACM SIGGRAPH Symposium on Computer Animation, pages 119-128, 2010. 2

[8] H. Hamacher, K. Leiner, and S. Ruzika. Quickest Cluster Flow Problems. In Peacock et al. [36], pages 327-336. 2

[9] D. Helbing and A. Johansson. Pedestrian, Crowd and Evacuation Dynamics. In Meyers [33], page 6476. 1

[10] D. Helbing and P. Molnar. Social force model for pedestrian dynamics. Phys. Rev. E, 51:4282-4286, 1995. 1

[11] M. Höcker, V. Berkhahn, A. Kneidl, A. Borrmann, and W. Klein. Graph-based approaches for simulating pedestrian dynamics in building models. In 8th European Conference on Product \& Process Modelling (ECPPM), University College Cork, Cork, Ireland, 2010. 2

[12] S. Hoogendoorn and P. Bovy. Pedestrian route-choice and activity scheduling theory and models. Transportation Research Part B, 38(2):169-190, 2004. 2

[13] R. Hughes. A continuum theory for the flow of pedestrians. Transportation Research Part B, 36(6):507-535, 2002. 2

[14] R. Hughes. The flow of human crowds. Annual Review of Fluid Mechanics, 35:169-182, 2003. 2

[15] H. Iwata. Walking about Virtual Environments on an Infinite Floor. In Proceedings of the IEEE Virtual Reality Conference, pages 286 - 293, Houston, TX, USA, 1999. 2

[16] H. Iwata, H. Yano, H. Fukushima, and H. Noma. CirculaFloor. IEEE Computer Graphics and Applications, 25(1):64-67, 2005. 2

[17] W.-K. Jeong and R. Whitaker. A fast eikonal equation solver for parallel systems. In SIAM conference on Computational Science and Engineering, 2007. 2

[18] W.-K. Jeong and R. Whitaker. A Fast Iterative Method for a Class of Hamilton-Jacobi Equations on Parallel Systems. Technical Report UUCS-07-010, University of Utah, School of Computing, 2007. 2 
[19] W.-K. Jeong and R. Whitaker. A Fast Iterative Method for Eikonal Equations. SIAM Journal on Scientific Computing, 30(5), 2008. 2

[20] A. Johansson, D. Helbing, and P. Shukla. Specification of the Social Force Pedestrian Model by Evolutionary Adjustment to Video Tracking Data. Advances in Complex Systems, 10(4):271-288, 2007. 1

[21] A. Kemloh Wagoum, A. Seyfried, and S. Holl. Modelling dynamic route choice of pedestrians to assess the criticality of building evacuation. arXiv eprint, 2011. submitted. 2

[22] R. Kimmel and J. Sethian. Computing geodesic paths on manifolds. In Proc. Natl. Acad. Sci. USA, pages 8431-8435, 1998. 2

[23] E. Kirik, T. Yurgel'yan, and D. Krouglov. The shortest time and/or the shortest path strategies in a CA FF pedestrian dynamics model. Journal of Siberian Federal University. Mathematics \& Physics, 2(3):271-278, 2009. 2

[24] E. Kirik, T. Yurgel'yan, and D. Krouglov. On realizing the shortest time strategy in a CA FF pedestrian dynamics model. Cybernetics and Systems, 42(1):1-15, 2011. 2

[25] W. Klingsch, C. Rogsch, A. Schadschneider, and M. Schreckenberg, editors. Pedestrian and Evacuation Dynamics 2008. Springer Berlin Heidelberg, 2010. 7

[26] T. Kretz. Pedestrian Traffic: on the Quickest Path. Journal of Statistical Mechanics: Theory and Experiment, P03012, 2009. 2

[27] T. Kretz. The use of dynamic distance potential fields for pedestrian flow around corners. In First International Conference on Evacuation Modeling and Management. TU Delft, 2009. 2

[28] T. Kretz. The Dynamic Distance Potential Field in a Situation with Asymmetric Bottleneck Capacities. In S. Bandini, S. Manzoni, H. Umeo, and G. Vizzari, editors, Cellular Automata - 9th International Conference on Cellular Automata for Research and Industry, ACRI2010. Springer Berlin / Heidelberg, 2010. 2

[29] T. Kretz. Applications of the Dynamic Distance Potential Field Method. In S. Dai et al. [42]. in press. 2

[30] T. Kretz, A. Große, S. Hengst, L. Kautzsch, A. Pohlmann, and P. Vortisch. Quickest Paths in Simulations of Pedestrians. Advances in Complex Systems, 2011. arXiv:1107.2004 [physics.soc-ph]. 1

[31] T. Kretz, S. Hengst, and P. Vortisch. Pedestrian Flow at Bottlenecks - Validation and Calibration of VISSIM's Social Force Model of Pedestrian Traffic and its Empirical Foundations. In M. Sarvi, editor, International Symposium of Transport Simulation 2008 (ISTS08), page electronic publication, Gold Coast, Australia, 2008. Monash University. 1

[32] G. Lämmel, M. Rieser, and K. Nagel. Large scale microscopic evacuation simulation. In Klingsch et al. [25], pages 547-553. 2

[33] R. Meyers, editor. Encyclopedia of Complexity and Systems Science. Springer, New York, 2009. 6, 7

[34] M. Moussaïd, D. Helbing, and G. Theraulaz. How simple rules determine pedestrian behavior and crowd disasters. Proceedings of the National Academy of Sciences, 108(17):6884, 2011. 2
[35] N. Nitzsche, U. D. Hanebeck, and G. Schmidt. Motion compression for telepresent walking in large target environments. Presence, 13(1):44-60, 2004. 2

[36] R. Peacock, E. Kuligowski, and J. Averill, editors. Pedestrian and Evacuation Dynamics. Springer, 2011. 6, 7

[37] A. Pérez Arias, U. Hanebeck, P. Ehrhardt, S. Hengst, T. Kretz, and P. Vortisch. Extended Range Telepresence for Pedestrian Simulations. In Peacock et al. [36], pages 199208. 3

[38] A. Pérez Arias, T. Kretz, P. Ehrhardt, S. Hengst, P. Vortisch, and U. Hanebeck. A Framework for Evaluating the VISSIM Traffic Simulation with Extended Range Telepresence. In Proceedings of the 22nd Annual Conference on Computer Animation and Social Agents (CASA 2009), Amsterdam, the Netherlands. University of Twente/CTIT, Twente, 2009. 3

[39] PTV. VISSIM 5.30 User Manual. PTV Planung Transport Verkehr AG, Stumpfstraße 1, D-76131 Karlsruhe, 2010. 1

[40] C. Rogsch and W. Klingsch. Basics of Software-Tools for Pedestrian MovementIdentification and Results. Fire Technology, pages 1-21, 2010. 2

[41] P. Rößler, U. Hanebeck, and N. Nitzsche. Feedback controlled motion compression for extended range telepresence. In Proceedings of IEEE Mechatronics \& Robotics (MechRob 2004), Special Session on Telepresence and Teleaction, pages 1447-1452, 2004. 2

[42] S. Dai et al., editor. Traffic and Granular Flow '09. Springer Berlin Heidelberg, 2011. in press. 7

[43] A. Schadschneider, W. Klingsch, H. Klüpfel, T. Kretz, C. Rogsch, and A. Seyfried. Evacuation Dynamics: Empirical Results, Modeling and Applications. In Meyers [33], page 3142.1

[44] A. Schomborg, K. Nökel, and A. Seyfried. Evacuation Assistance for a Sports Arena Using a Macroscopic Network Model. In Peacock et al. [36], pages 389-398. 2

[45] M. C. Schwaiger, T. Thummel, and H. Ulbrich. A 2DMotion Platform: The Cybercarpet. In Proceedings of Second Joint EuroHaptics Conference and Symposium on Haptic Interfaces for Virtual Environment and Teleoperator Systems, pages $415-420,2007.2$

[46] J. Shopf, C. Oat, and J. Barczak. GPU Crowd Simulation. ACM Transactions on Graphics, Siggraph Asia 2008, 27:0, 2008. 2

[47] B. Steffen and A. Seyfried. Modeling of pedestrian movement around 90 and 180 degree bends. In Proc. of Workshop on Fire Protection and Life Safety in Buildings and Transportation Systems, 2009. 2

[48] R. Stemmer, R. Brockers, S. Drüe, and J. Thiem. Comprehensive Data Acquisition for a Telepresence Application. In Proceedings of Systems, Man, and Cybernetics, pages 53445349, The Hague, The Netherlands, 2004. 2

[49] A. Treuille, S. Cooper, and Z. Popović. Continuum crowds. In ACM SIGGRAPH 2006 Papers, page 1168, 2006. 2

[50] J. Yoon, J. Park, and J. Ryu. Walking Control of a DualPlanar Parallel Robot for Omni-Directional Locomotion Interface. In Proceedings of IEEE/RSJ International Conference on Intelligent Robots and Systems, pages 1151 - 1156, 2005. 2 